\title{
LHC Run I bounds on minimal lepton flavour violation in Type-III see-saw: a case study
}

\author{
Nuno Rosa Agostinho, ${ }^{a}$ O.J.P. Éboli ${ }^{b}$ and M.C. Gonzalez-Garcia ${ }^{a, c, d}$ \\ ${ }^{a}$ Departament de Fisíca Quàntica i Astrofísica and Institut de Ciencies del Cosmos, \\ Universitat de Barcelona, Diagonal 647, Barcelona, E-08028 Spain \\ ${ }^{b}$ Instituto de Física, Universidade de São Paulo, \\ Rua do Matão 1371, São Paulo, SP, Brazil \\ ${ }^{c}$ Institució Catalana de Recerca i Estudis Avançats (ICREA), \\ Pg. Lluis Companys 23, Barcelona, 08010 Spain \\ ${ }^{d}$ C.N. Yang Institute for Theoretical Physics, State University of New York at Stony Brook, \\ Stony Brook, NY, 11794-3840 U.S.A. \\ E-mail: nunorosaagostinho@ub.edu, eboli@if.usp.br, \\ maria.gonzalez-garcia@stonybrook.edu
}

ABSTRACT: In minimal lepton flavour violation Type-III see-saw models the flavour structure of the couplings of the triplet fermions to the Standard Model leptons can be reconstructed from the neutrino mass matrix and lepton number violation is very suppressed. Here, we explore the possibility of imposing bounds on this class of models with results of existing LHC searches and we quantify how the information on flavour and charge of the produced leptons is important for maximal sensitivity. With this aim we obtain the bounds which can be derived from the Run I search for events which contain two charged leptons (either electron or muons of equal or opposite sign), two jets from a hadronically decaying $W$ boson and large missing transverse momentum. We find that using the information on charge and flavour of the leptons in the above final state it is possible to unambiguously rule out this scenario with triplet masses lighter than $300 \mathrm{GeV}$ at $95 \% \mathrm{CL}$. The same analysis allows to exclude triplet masses up to $480 \mathrm{GeV}$ at $95 \%$ CL for normal ordering of neutrino masses and specific values of a Majorana CP phase currently undetermined by neutrino physics. We also show that it is not possible to put an unambiguous bound on the mass of the new states if the flavor and charge information is not condsidered.

Keywords: Beyond Standard Model, Neutrino Physics

ARXIV EPRINT: 1708.08456 


\section{Contents}

1 Introduction 1

2 MLFV Type-III see-saw model 3

3 Case study: $p p \rightarrow l l^{\prime} j j \nu \nu+9$

3.1 Contributing subprocesses 9

$\begin{array}{lll}3.2 & \text { Simulation of the expected event rates } & 10\end{array}$

4 Analysis: results and discussion $\quad 12$

\section{Introduction}

Massive neutrinos are our first and only undoubted evidence of physics beyond the Standard Model (SM). The evidence arises from a variety of neutrino experiments which have detected the effect of their mass via the flavour oscillation of neutrinos with energies ranging between tens of $\mathrm{keV}$ and tens of $\mathrm{GeV}[1]$. The obvious question that this observation raises is that of the dynamics of the New Physics (NP) responsible for the neutrino mass.

It is well known that in the framework of effective operators for NP there is just one dimension-five operator which can be built [2], $\mathcal{O}=\alpha_{5} / \Lambda_{\mathrm{LN}} L_{L} L_{L} H H$, where $L_{L}$ and $H$ are the leptonic and Higgs $\mathrm{SU}(2)_{L}$ doublets. This operator breaks total lepton number and after electroweak symmetry breaking it generates Majorana masses for the neutrinos $m_{\nu} \sim \alpha_{5} v^{2} / \Lambda_{\mathrm{LN}}$, where $v$ is the SM Higgs vacuum expectation value (vev). This explains the lightness of the neutrino mass due to the large scale of total lepton number violation $\Lambda_{\mathrm{LN}}$. In the simplest UV completions, this dimension-5 operator can be generated by the tree-level exchange of three types of new states: lepton singlets in the Type-I see-saw scenarios [3-6], a scalar triplet for the Type-II mechanisms [7-11], and lepton triplets for Type-III models [12]. In any of these mechanisms the smallness of the neutrino mass can be naturally explained with Yukawa couplings $\lambda \sim \mathcal{O}(1)$ if the masses of the new states are $M \sim \Lambda_{\mathrm{LN}} \sim 10^{14-15} \mathrm{GeV}$. Notwithstanding consistent models of lower scale see-saw exist in the literature for some time; see e.g. [13-15].

Given the energy range of the neutrino experiments it is clear that if the NP scale is beyond $\sim \mathrm{GeV}$ it is not possible to clarify its origin within the oscillation neutrino experiments themselves. On the other hand, the high energy frontier is currently being explored by the CERN Large Hadron Collider (LHC) which has been running for eight years with a reach to NP at the TeV scale. So far no clear evidence of NP has been observed at LHC which bears implications for models constructed to explain the neutrino masses containing new states at the $\mathrm{TeV}$ scale. 
Generically the expected event rates at LHC in neutrino mass models depend not only on the mass and weak charge of the new states involved, but also on the flavour structure of their couplings which determines their decay modes. A priory, the decay channels are arbitrary in most Type-I and Type-III see-saw models, making difficult to derive unambiguous constraints on the NP scale in these scenarios [16, 17]. For example searches for triplet leptons of the Type-III see-saw models have been performed both by CMS [18-21] and ATLAS [22, 23] collaborations, however, most of these searches have been carried out within the context of simplified models such as ref. [24] and the derived bounds can be evaded depending on which is the dominant decay mode of the triplet leptons.

One exception are see-saw models which extend the principle of minimal flavour violation to the leptonic sector. Minimal flavour violation was first introduced for quarks [25-27] as a way to explain the absence of NP effects in flavour changing processes in meson decays. The basic assumption is that the only source of flavour mixing in the full theory is the same as in the SM, i.e. the quark Yukawa couplings. This idea was latter on extended for leptons $[28,29]$ and in particular to TeV scale see-saw models [28-31].

From the point of view of LHC phenomenology minimal lepton flavour violation (MLFV) see-saw models are attractive since, a) the new states can be light enough to be produced at LHC, and b) their signatures are fully determined by the neutrino parameters. As discussed in ref. [30] scalar (Type-II) see-saw with light doublet-triplet mixing is a light scale MLFV model by construction (for early study of their observability see for example [32, 33]). In ref. [30] simple MLFV models for fermionic see-saw were also presented. In Type-I see-saw the new states are singlets under the SM gauge group, and therefore, they can only be produced via their mixing with the SM neutrinos. This leads to small production rates which makes the model only marginally testable at LHC. Type-III seesaw fermions, on the contrary, are $\mathrm{SU}(2)_{L}$ triplets with weak-interaction pair-production cross section, and consequently, having the potential to allow for tests of the hypothesis of MLFV.

In ref. [34] we studied the potential of LHC to unravel the existence of triplet fermionic states that appear in MLFV Type-III see-saw models of neutrino mass. Here, we obtain the bounds on the NP scale of this scenario that originates from the ATLAS [22] searches in the final state topology containing two charged leptons (electrons and/or muons), two jets compatible with a hadronically decaying $W$ and missing transverse momentum. This ATLAS analysis is best suited for testing the MLFV scenario because it classifies the final states in the different flavour and charge combinations, allowing to fully exploit the predictions of the MLFV Type-III see-saw model. We find that using this ATLAS data it is possible to unambiguously rule out the MLFV Type-III see-saw scenario with triplet masses lighter than $300 \mathrm{GeV}$ at $95 \% \mathrm{CL}$ irrespective of the neutrino mass ordering and other unknowns in the light neutrino sector, in particular of the so-far undetermined Majorana $\mathrm{CP}$ violating phase. The same analysis allows to rule out triplet masses up to $480 \mathrm{GeV}$ at $95 \% \mathrm{CL}$ for normal order (NO) scenarios and specific values of that phase. We also obtained the limits neglecting the flavour and charge information and we show that for a large range of the Majorana $\mathrm{CP}$ violating phase for normal order scenario it is not possible to obtain any constraints on the mass of the new triplet states. 
This paper is organized as follows. We first summarize in section 2 the basics of the MLFV Type-II see-saw model and we quantify the allowed range of the relevant couplings controlling the triplet decay modes as derived from the present analysis of neutrino oscillation data from ref. [35]. Section 3 describes our simulation of signal events by the reaction $p p \rightarrow l l^{\prime} j j \nu \nu$ with $l^{(\prime)}=e$ or $\mu$ in the context of the MLFV Type-III see-saw model. The quantification of the bounds is presented in section 4. In doing so we have put special emphasis and we have quantified how the information on flavour and charge of the produced leptons is important for maximal sensitivity to MLFV.

\section{MLFV Type-III see-saw model}

In ref. [34] we introduced the simplest MLFV Type-III see-saw model which was adapted from the Type-I one presented in ref. [30]. For completeness we summarize here its main features.

The particle contents of model is that of the SM extended with two fermion triplets $\vec{\Sigma}=\left(\Sigma_{1}, \Sigma_{2}, \Sigma_{3}\right)$ and $\vec{\Sigma}^{\prime}=\left(\Sigma_{1}^{\prime}, \Sigma_{2}^{\prime}, \Sigma_{3}^{\prime}\right)$, each one formed by three right-handed Weyl spinors of zero hypercharge. Hence, the Lagrangian is

$$
\mathcal{L}=\mathcal{L}_{\mathrm{SM}}+\mathcal{L}_{K}+\mathcal{L}_{Y}+\mathcal{L}_{\Lambda}
$$

with

$$
\begin{aligned}
& \mathcal{L}_{K}=i\left(\overline{\vec{\Sigma}} \not D_{\mu} \vec{\Sigma}+\overline{\vec{\Sigma}^{\prime}} \not D_{\mu} \vec{\Sigma}^{\prime}\right) \\
& \mathcal{L}_{Y}=-Y_{\beta}^{\dagger} \overline{L_{L \beta}^{w}}(\vec{\Sigma} \cdot \vec{\tau}) \tilde{\phi}-\epsilon Y_{\beta}^{\prime \dagger} \overline{L_{L \beta}^{w}}\left(\vec{\Sigma}^{\prime} \cdot \vec{\tau}\right) \tilde{\phi}+\text { h.c. } \\
& \mathcal{L}_{\Lambda}=-\frac{\Lambda}{2}\left(\overline{\overrightarrow{\Sigma^{c}}} \vec{\Sigma}^{\prime}+\overline{\overrightarrow{\Sigma^{\prime}}} \vec{\Sigma}\right)-\frac{\mu}{2} \overline{\vec{\Sigma}^{\prime}} \vec{\Sigma}^{\prime}-\frac{\mu^{\prime}}{2} \overrightarrow{\vec{\Sigma} c} \vec{\Sigma}+\text { h.c. }
\end{aligned}
$$

Here $\vec{\tau}$ are the Pauli matrices, the gauge covariant derivative is defined as $D_{\mu}=\partial_{\mu}+i g \vec{T}$. $\vec{W}_{\mu}$, where $\vec{T}$ are the three-dimensional representation of the $\mathrm{SU}(2)_{L}$ generators, $\phi$ stands for the SM Higgs doublet field, and $L_{\beta}^{w}=\left(\nu_{\beta}^{w}, \ell_{\beta}^{w}\right)^{T}$ are the three weak eigenstate lepton doublets of the SM. The parameters $\epsilon, \mu$ and $\mu^{\prime}$ are flavour-blind and small, i.e., the scales $\mu$ and $\mu^{\prime}$ are much smaller than $\Lambda$ and $v$ while $\epsilon \ll 1$.

The Lagrangian in eq. (2.1) breaks total lepton number due to the simultaneous presence of the Yukawa terms $Y_{i}$ and $\epsilon Y_{i}^{\prime}$ as well as to the existence of the $\mu$ and $\mu^{\prime}$ terms. Thus in the limit $\mu, \mu^{\prime}, \epsilon \rightarrow 0$ it is possible to define a conserved total lepton number by assigning $L\left(L^{w}\right)=L(\Sigma)=-L\left(\Sigma^{\prime}\right)=1$. Without any loss of generality one can work in a basis in which $\Lambda$ is real while both $Y$ and $Y^{\prime}$ are complex. In general the parameters $\mu$ and $\mu^{\prime}$ would be complex, but for the sake of simplicity we take them to be real in what follows though it is straight forward to generalize the expression to include the relevant phases [36]. 
After electroweak symmetry breaking, in the unitary gauge, the leptonic mass matrices are

$$
\mathcal{L}_{m}=-\frac{1}{2}\left(\overline{\vec{\nu}_{L}^{c}} \overline{\tilde{N}_{R}} \overline{\tilde{N}_{R}^{\prime}}\right) M_{0}\left(\begin{array}{c}
\nu_{L}^{\vec{w}} \\
\tilde{N}_{R}^{c} \\
\tilde{N}_{R}^{\prime c}
\end{array}\right)-\left(\overline{\overrightarrow{\ell_{L}^{w}}} \overline{E_{L}} \overline{E_{L}^{\prime}}\right) M_{ \pm}\left(\begin{array}{c}
\overrightarrow{\ell_{R}^{w}} \\
E_{R} \\
E_{R}^{\prime}
\end{array}\right)+\text { h.c. }
$$

with

$$
M_{0}=\left(\begin{array}{ccc}
0 & \frac{v}{\sqrt{2}} Y^{T} & \epsilon \frac{v}{\sqrt{2}} Y^{\prime T} \\
\frac{v}{\sqrt{2}} Y & \mu^{\prime} & \Lambda \\
\epsilon \frac{v}{\sqrt{2}} Y^{\prime} & \Lambda & \mu
\end{array}\right) \quad \text { and } \quad M_{ \pm}=\left(\begin{array}{ccc}
\frac{v}{\sqrt{2}} Y^{\ell} & v Y^{\dagger} & \epsilon v Y^{\prime \dagger} \\
0 & \mu^{\prime} & \Lambda \\
0 & \Lambda & \mu
\end{array}\right)
$$

where $Y^{\ell}$ are the charged lepton Yukawa couplings of the SM. $\vec{\nu}^{w}$ and $\vec{\ell}^{w}$ are column vectors containing respectively the three neutrinos and charged leptons of the SM in the weak basis. The charge eigenstates Dirac fermions $E$ and $E^{\prime}$ and the neutral Majorana fermions $\tilde{N}$ and $\tilde{N}^{\prime}$ are defined in terms of the triplet states, $\Sigma_{ \pm}^{(\prime)}=\frac{1}{\sqrt{2}}\left(\Sigma_{1}^{(\prime)} \mp i \Sigma_{2}^{(\prime)}\right)$, and $\Sigma_{0}^{(\prime)}=\Sigma_{3}^{(\prime)}$, as

$$
E^{(\prime)}=\Sigma_{-}^{(\prime)}+\Sigma_{+}^{(\prime)^{c}} \quad \tilde{N}^{(\prime)}=\Sigma_{0}^{(\prime)}+\Sigma_{0}^{(\prime)^{c}}
$$

The mass basis is composed of:

- Three light Majorana neutrinos $\nu_{i}$ (with the lightest one being massless) and three light charged leptons $\ell_{i}$ with masses

$$
\begin{aligned}
& m_{\nu}^{\mathrm{diag}}=V^{\nu T}\left[-\frac{v^{2}}{2 \Lambda} \epsilon\left[\left(Y^{\prime}-\frac{1}{\epsilon} \frac{\mu}{2 \Lambda} Y\right)^{T} Y+Y^{T}\left(Y^{\prime}-\frac{1}{\epsilon} \frac{\mu}{2 \Lambda} Y\right)\right]\right] V^{\nu}, \\
& m_{\ell}^{\mathrm{diag}}=\frac{v}{\sqrt{2}} V_{R}^{\ell^{\dagger}} Y^{\ell \dagger}\left[1-\frac{v^{2}}{2 \Lambda^{2}} Y^{\dagger} Y\right] V_{L}^{\ell},
\end{aligned}
$$

where $V^{\nu}$ and $V_{L, R}^{\ell}$ being $3 \times 3$ unitary matrices and in general, one can choose the flavour basis such that $V_{L}^{\ell}=V_{R}^{\ell}=I$.

- Two charged heavy leptons, $E_{1}^{-}$and $E_{2}^{+}$, both with masses $M \simeq \Lambda\left(1 \mp \frac{\mu+\mu^{\prime}}{2 \Lambda}\right)$.

- Two heavy Majorana neutral leptons and two charged heavy leptons also with masses $M \simeq \Lambda\left(1 \mp \frac{\mu+\mu^{\prime}}{2 \Lambda}\right)$ with which we build a quasi-Dirac heavy state $N$.

The relation between the weak and mass eigenstate to first order in the small parameters $\mu, \mu^{\prime}$ and $\epsilon$ is:

$$
\begin{aligned}
& \nu_{L}^{w}=V^{\nu} \nu_{L}+\frac{v}{\sqrt{2} \Lambda} Y^{\dagger} N_{L}+\frac{v}{\sqrt{2} \Lambda}\left(\epsilon Y^{\prime \dagger}-\left(\frac{3 \mu+\mu^{\prime}}{4 \Lambda}\right) Y^{\dagger}\right) N_{R}^{c}, \\
& \ell_{L}^{w}=\ell_{L}+\frac{v}{\Lambda} Y^{\dagger} E_{1 L}^{-}+\frac{v}{\Lambda}\left(\epsilon Y^{\prime \dagger}-\left(\frac{3 \mu+\mu^{\prime}}{4 \Lambda}\right) Y^{\dagger}\right) E_{2 R}^{+c},
\end{aligned}
$$




$$
\begin{aligned}
& \ell_{R}^{w}=\ell_{R} \\
& \tilde{N}_{L}=N_{R}^{c}-\left(\frac{\mu-\mu^{\prime}}{4 \Lambda}\right) N_{L}-\frac{v}{\sqrt{2} \Lambda}\left(\epsilon Y^{\prime}-\frac{\mu}{\Lambda} Y\right) V^{\nu} \nu_{L}, \\
& \tilde{N}_{L}^{\prime}=N_{L}+\left(\frac{\mu-\mu^{\prime}}{4 \Lambda}\right) N_{R}^{c}-\frac{v}{\sqrt{2} \Lambda} Y V^{\nu} \nu_{L}, \\
& E_{L}=E_{2 R}^{+c}-\left(\frac{\mu-\mu^{\prime}}{4 \Lambda}\right) E_{1 L}^{-}-\frac{v}{\Lambda}\left(\epsilon Y^{\prime}-\frac{\mu}{\Lambda} Y\right) \ell_{L}, \\
& E_{R}=E_{1 R}^{-}-\left(\frac{\mu-\mu^{\prime}}{4 \Lambda}\right) E_{2 L}^{+c}, \\
& E_{L}^{\prime}=E_{1 L}^{-}+\left(\frac{\mu-\mu^{\prime}}{4 \Lambda}\right) E_{2 R}^{+c}-\frac{v}{\Lambda} Y \ell_{L}, \\
& E_{R}^{\prime}=E_{2 L}^{+c}+\left(\frac{\mu-\mu^{\prime}}{4 \Lambda}\right) E_{1 R}^{-} .
\end{aligned}
$$

From the above relations it follows that the neutral weak interactions of the light states take the same form as that on the SM and the charged current interactions involve a $3 \times 3$ unitary matrix $U_{\mathrm{LEP}}=V^{\nu}$ which after phase redefinition of the light charged leptons, can be chosen

$$
U_{\mathrm{LEP}}=\left(\begin{array}{ccc}
1 & 0 & 0 \\
0 & c_{23} & s_{23} \\
0 & -s_{23} & c_{23}
\end{array}\right)\left(\begin{array}{ccc}
c_{13} & 0 & s_{13} e^{-i \delta_{\mathrm{CP}}} \\
0 & 1 & 0 \\
-s_{13} e^{i \delta_{\mathrm{CP}}} & 0 & c_{13}
\end{array}\right)\left(\begin{array}{ccc}
c_{21} & s_{12} & 0 \\
-s_{12} & c_{12} & 0 \\
0 & 0 & 1
\end{array}\right)\left(\begin{array}{ccc}
e^{-i \alpha} & 0 & 0 \\
0 & e^{i \alpha} & 0 \\
0 & 0 & 1
\end{array}\right),
$$

where $c_{i j} \equiv \cos \theta_{i j}$ and $s_{i j} \equiv \sin \theta_{i j}$. The angles $\theta_{i j}$ can be taken without loss of generality to lie in the first quadrant, $\theta_{i j} \in[0, \pi / 2]$ and the phases $\delta_{\mathrm{CP}}, \alpha \in[0,2 \pi]$. The leptonic mixing matrix contains only one Majorana phase because there are only two heavy triplets and consequently only two light neutrinos are massive while the lightest one remains massless. Also notice that unitarity violation in the charged current and flavour mixing in the neutral current of the light leptons in generated at higher order [36-38].

This model is MLFV because one can fully reconstruct the neutrino Yukawa coupling $Y$ and the combination $\widehat{Y^{\prime}}=Y^{\prime}-\frac{1}{\epsilon} \frac{\mu}{2 \Lambda} Y$ from the neutrino mass matrix [30] up to two real normalization factors $y$ and $\hat{y}^{\prime}$ as follows:

\begin{tabular}{|c|c|}
\hline $\mathrm{NO}\left(m_{1}=0<m_{2}<m_{3}\right)$ & $\mathrm{IO}\left(m_{3}=0<m_{1}<m_{2}\right)$ \\
\hline$r=\frac{\Delta m_{21}^{2}}{\Delta m_{32}^{2}}$ & $r=-\frac{\Delta m_{21}^{2}}{\Delta m_{31}^{2}}$ \\
$\rho=\frac{\sqrt{1+r}-\sqrt{r}}{\sqrt{1+r}+\sqrt{r}}$ & $\rho=\frac{\sqrt{1+r}-1}{\sqrt{1+r}+1}$ \\
$m_{2,3}=\frac{\epsilon y \widehat{y}^{\prime}}{\Lambda}(1 \mp \rho)$ & $m_{1,2}=\frac{\epsilon y \widehat{y}^{\prime} v^{2}}{\Lambda}(1 \mp \rho)$ \\
$Y_{\beta}=\frac{y}{\sqrt{2}}\left(\sqrt{1+\rho} U_{\beta 3}^{*}+\sqrt{1-\rho} U_{\beta 2}^{*}\right)$ & $Y_{\beta}=\frac{y}{\sqrt{2}}\left(\sqrt{1+\rho} U_{\beta 2}^{*}+\sqrt{1-\rho} U_{\beta 1}^{*}\right)$ \\
$\widehat{Y^{\prime}}{ }_{\beta}=\frac{\widehat{y^{\prime}}}{\sqrt{2}}\left(\sqrt{1+\rho} U_{\beta 3}^{*}-\sqrt{1-\rho} U_{\beta 2}^{*}\right)$ & $\widehat{Y^{\prime}}=\frac{\widehat{y^{\prime}}}{\sqrt{2}}\left(\sqrt{1+\rho} U_{\beta 2}^{*}-\sqrt{1-\rho} U_{\beta 1}^{*}\right)$ \\
\hline
\end{tabular}


In writing eq. (2.20) we follow the notation of using greek index $\beta$ to denote lepton flavour index $\beta, \gamma=e, \mu, \tau$. And in what follows we will use roman index $i, j=1,2,3$ to denote the light neutrino mass index.

We plot in figure 1 the ranges of the Yukawa couplings $\left|\tilde{Y}_{e}\right|^{2} \equiv\left|Y_{e}\right|^{2} / y^{2}$ and $\left|\tilde{Y}_{\mu}\right|^{2} \equiv$ $\left|Y_{\mu}\right|^{2} / y^{2}$ obtained by projecting the allowed ranges of oscillation parameters from the global analysis of neutrino data [35] using eqs. (2.20). In the first and second rows we plot the ranges of the Yukawa couplings as a function of the unknown Majorana phase $\alpha$ while the lower row shows the correlation between the electron and muon Yukawa couplings. This figure illustrates the quite different allowed ranges and correlation of the electron and muon Yukawa couplings in the two orderings. As a curiosity we notice that in NO the dependence of the range of $\left|Y_{e}\right|$ on $\alpha$ is driven by the present hint of $\delta_{\mathrm{CP}} \sim 270^{\circ}$ in the oscillation data analysis [35] because $\alpha$ enters via $\operatorname{Re}\left(U_{e 3} U_{e 2}^{*}\right) \propto \cos \left(\alpha+\delta_{\mathrm{CP}}\right)$.

In what respects the interactions of the heavy states, as discussed in ref. [34], lepton number violating couplings appear due to $\mathcal{O}\left(\epsilon, \mu / \Lambda, \mu^{\prime} / \Lambda\right)$ mixings and mass splittings in the heavy states, that are, by hypothesis, very suppressed in MLFV models. This renders the lepton number violating processes involving heavy fermions unobservable at LHC, at a difference with the non MLFV scenarios for Type-III see-saw for which $\Delta L=2$ final states constitute a smoking gun $[39,40]$. Consequently in what follows we concentrate in the lepton conserving interaction Lagrangian with $\Lambda=M$ being the common mass of the heavy states:

$$
\begin{aligned}
\mathcal{L}_{W}= & -g\left(\overline{E_{1}^{-}} \gamma^{\mu} N W_{\mu}^{-}-\bar{N} \gamma^{\mu} E_{2}^{+} W_{\mu}^{-}\right)+\text {h.c. } \\
& -g\left(\frac{1}{\sqrt{2}} K_{\beta} \overline{\ell_{\beta L}} \gamma^{\mu} N_{L} W_{\mu}^{-}+\overline{K_{i} \overline{\nu_{i L}}} \gamma^{\mu} E_{2 L}^{+} W_{\mu}^{-}\right)+\text {h.c. } \\
\mathcal{L}_{Z}= & g C_{W}\left(\overline{E_{1}^{-}} \gamma^{\mu} E_{1}^{-} Z_{\mu}-\overline{E_{2}^{+}} \gamma^{\mu} E_{2}^{+} Z_{\mu}\right) \\
& +\frac{g}{2 \sqrt{2} C_{W}}\left(\frac{1}{\sqrt{2}} \overline{K_{i}} \overline{\nu_{i L}} \gamma^{\mu} N_{L} Z_{\mu}+K_{\beta} \overline{\ell_{\beta L}} \gamma^{\mu} E_{1 L}^{-} Z_{\mu}\right)+\text { h.c. } \\
\mathcal{L}_{\gamma}= & e\left(\overline{E_{1}^{-}} \gamma^{\mu} E_{1}^{-} A_{\mu}-\overline{E_{2}^{+}} \gamma^{\mu} E_{2}^{+} A_{\mu}\right) \\
\mathcal{L}_{h^{0}}= & \frac{g M}{\sqrt{2} M_{W}}\left(\frac{1}{\sqrt{2}} \overline{K_{i}} \overline{\nu_{i L}} N_{R}+K_{\beta} \overline{\ell_{\beta L}} E_{1 R}^{-}\right)+\text {h.c. . }
\end{aligned}
$$

where $c_{W}$ stands for the cosine of the weak mixing angle and the lepton number conserving couplings of the heavy triplet fermions are

$$
K_{\beta}=-\frac{v}{\sqrt{2} M} Y_{\beta}^{*}, \quad \bar{K}_{i}=\sum_{\beta=e, \mu, \tau}\left(U_{\mathrm{LEP}}^{*}\right)_{\beta i} K_{\beta},
$$

which verify

$$
\sum_{\beta=e, \mu, \tau}\left|K_{\beta}\right|^{2}=\sum_{i=1}^{3}\left|\bar{K}_{i}\right|^{2}=\frac{y^{2} v^{2}}{2 M^{2}} .
$$

Notice that flavour structures of the couplings $K$ and $\bar{K}$ are fully determined by the low energy neutrino parameters and fully determined by the oscillation data up to the Majorana 

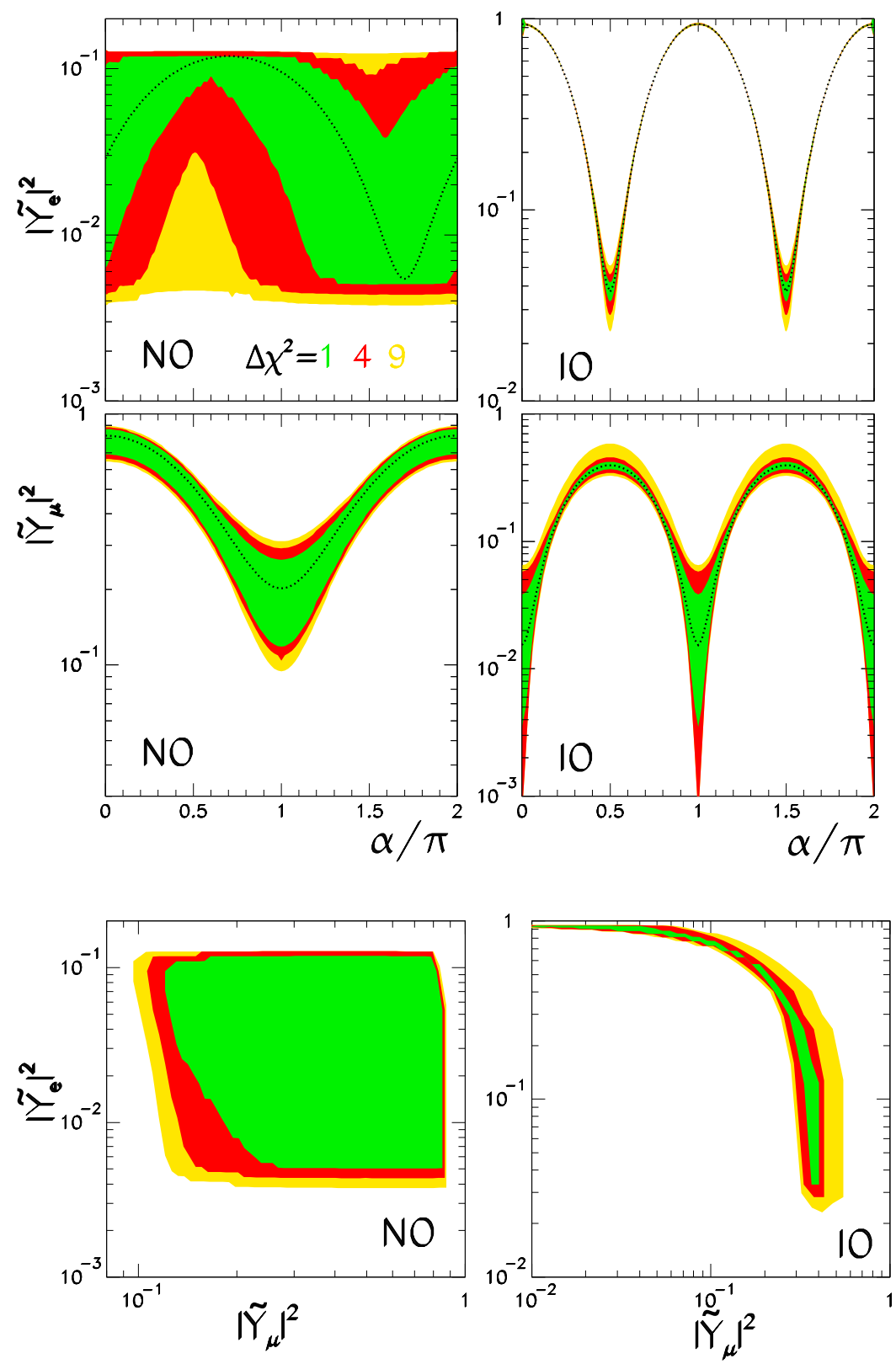

Figure 1. Allowed ranges of the Yukawa couplings $\left|\tilde{Y}_{e}\right|^{2} \equiv\left|Y_{e}\right|^{2} / y^{2}$ and $\left|\tilde{Y}_{\mu}\right|^{2} \equiv\left|Y_{\mu}\right|^{2} / y^{2}$ obtained from the global analysis of neutrino data in ref. [35]. The upper four panel shows the values of the couplings as a function of the unknown Majorana phase $\alpha$. The correlation between the two couplings is shown in the two lower panels. The left (right) panels correspond to normal (inverted) ordering. The dotted line corresponds to the best fit values. The ranges in the filled green, red and yellow areas are shown at $1 \sigma, 2 \sigma$, and $3 \sigma$ with 1 dof $\left(\Delta \chi^{2}=1,4,9\right.$ respectively). 
phase $\alpha$. Their strengths are, however, arbitrary as they are controlled by the normalization factor $y v / M$ while it is the combination $\epsilon y \widehat{y}^{\prime} / M$ what is fixed by the neutrino masses.

From these interactions we can obtain the decay widths of the heavy states [41]:

$$
\begin{aligned}
\Gamma\left(N \rightarrow \ell_{\beta}^{-} W^{+}\right) & =\frac{g^{2}}{64 \pi}\left|K_{\beta}\right|^{2} \frac{M^{3}}{M_{W}^{2}}\left(1-\frac{M_{W}^{2}}{M^{2}}\right)\left(1+\frac{M_{W}^{2}}{M^{2}}-2 \frac{M_{W}^{4}}{M^{4}}\right), \\
\Gamma\left(N \rightarrow \nu_{i} Z\right) & =\frac{g^{2}}{128 \pi c_{w}^{2}}\left|\bar{K}_{i}\right|^{2} \frac{M^{3}}{M_{Z}^{2}}\left(1-\frac{M_{Z}^{2}}{M^{2}}\right)\left(1+\frac{M_{Z}^{2}}{M^{2}}-2 \frac{M_{Z}^{4}}{M^{4}}\right), \\
\Gamma\left(N \rightarrow \nu_{i} h^{0}\right) & =\frac{g^{2}}{128 \pi}\left|\bar{K}_{i}\right|^{2} \frac{M^{3}}{M_{W}^{2}}\left(1-\frac{M_{h^{0}}^{2}}{M^{2}}\right)^{2}, \\
\Gamma\left(E_{2}^{+} \rightarrow \nu_{i} W^{+}\right) & =\frac{g^{2}}{32 \pi}\left|\bar{K}_{i}\right|^{2} \frac{M^{3}}{M_{W}^{2}}\left(1-\frac{M_{W}^{2}}{M^{2}}\right)\left(1+\frac{M_{W}^{2}}{M^{2}}-2 \frac{M_{W}^{4}}{M^{4}}\right), \\
\Gamma\left(E_{1}^{-} \rightarrow \ell_{\beta}^{-} Z\right) & =\frac{g^{2}}{64 \pi c_{W}^{2}}\left|K_{\beta}\right|^{2} \frac{M^{3}}{M_{Z}^{2}}\left(1-\frac{M_{Z}^{2}}{M^{2}}\right)\left(1+\frac{M_{Z}^{2}}{M^{2}}-2 \frac{M_{Z}^{4}}{M^{4}}\right), \\
\Gamma\left(E_{1}^{-} \rightarrow \ell_{\beta}^{-} h^{0}\right) & =\frac{g^{2}}{64 \pi}\left|K_{\beta}\right|^{2} \frac{M^{3}}{M_{W}^{2}}\left(1-\frac{M_{h^{0}}^{2}}{M^{2}}\right)^{2} .
\end{aligned}
$$

Therefore, using eq. (2.26) the total decay widths for the three triplet fermions $F=$ $N, E_{1}^{-}, E_{2}^{+}$are

$$
\Gamma_{F}^{\mathrm{TOT}}=\frac{g^{2} M^{3}}{64 \pi M_{W}^{2}} \frac{y^{2} v^{2}}{M^{2}}\left(1+\mathcal{F}_{F}(M)\right)
$$

where $\mathcal{F}_{F}(M) \rightarrow 0$ for $M \gg m_{h^{0}}, M_{Z}, M_{W}$. Consequently, the arbitrary $y$ factor cancels out in the branching ratios. Furthermore the branching ratio in a final state with a charge lepton of flavour $\alpha$ or a neutrino state $i$ produced in the vertex of the heavy state decay is proportional to $\left|\tilde{Y}_{\alpha}\right|^{2}$ and $\left|\sum_{\beta} \tilde{Y}_{\beta}\left(U_{\mathrm{LEP}}\right)_{\beta i}\right|^{2}$ times a kinematic factor depending solely on the heavy mass $M$.

Unlike in a general Type-III see-saw model for which the branching ratio of $N$ or $E_{i}^{ \pm}$into a light lepton of a given flavour can be negligibly small, for the MLFV TypeIII see-saw model the branching ratios in the different light lepton flavours are fixed by the neutrino physics and are non-vanishing as can be seen from figure 1 and eqs. (2.27). This makes the flavour composition of the final states in any decay chain of the heavy leptons to be fully determined given a neutrino mass ordering. Consequently in the narrow width approximation the only free parameters in the model are the mass of the states and the Majorana phase $\alpha$, making the model more unambiguously testable. Conversely, as discussed in ref. [34] in this MLFV Type-III model the values of the neutrino masses imply a lower bound on the total decay width of the triplet fermions as a consequence of the hierarchy between the $L$-conserving and $L$-violating $y$ and $\epsilon \widehat{y^{\prime}}$ constants. So their decay length is too short to produce a detectable displaced decay vertex signature $[42,43]$ at difference with other see-saw models [32, 39, 40, 44].

In order to simulate the expected signals in this MLFV Type-III see-saw model we have implemented the Lagrangian in eqs. (2.21)-(2.24) using the package FeynRules [45, 46]. We have made available the corresponding model files at the corresponding URL [47]. 


\section{Case study: $p p \rightarrow l l^{\prime} j j \nu \nu$}

In order to study the sensitivity of LHC Run I to MLFV Type-III see-saw signatures we will use the event topologies studied by ATLAS in ref. [22] which contain two charged leptons (either electron or muons), two jets from a hadronically decaying $W$ boson and large missing transverse momentum. ATLAS used these topologies to search for heavy fermions in the context of the simplified Type-III see-saw model as implemented in ref. [24]. They presented their results as number of events for the six different flavour and charge lepton pair combinations: same sign (SS) and opposite sign (SS) $e e, \mu \mu$ and $e \mu$. Using those they obtain bounds on the triplet mass which depend on the decay branching ratio into the different flavours. In particular for triplets decaying mostly into $\tau$ 's no bound can be derived.

On the contrary, as stressed in the previous section, in the MLFV scenario the flavour and lepton number of the final states produced in the heavy fermion decay chain is very much constrained. Thus having the final states classified in the different flavour and charge combinations makes the result in ref. [22] best suited for testing the MLFV scenario as we quantify next.

\subsection{Contributing subprocesses}

Let us start by listing the possible subprocesses contributing to the different flavour and charge combinations in the MLFV Type-III see-saw model. They all proceed by the production of a pair of heavy triplet states and their subsequent decay. The pair production of the fermion triplets takes place via gauge interactions, and, therefore, it depends exclusively upon the mass of the new states. On the other hand, the branching ratios of these fermions into the final states described in the previous section depend upon the Yukawa couplings $\tilde{Y}_{\beta}$ which vary in the different subprocesses:

- $p p \rightarrow E_{1}^{-} \tilde{N}, \quad\left(E_{1}^{-} \rightarrow l_{\beta}^{-} Z, Z \rightarrow \nu_{i} \nu_{i}\right) \quad\left(\tilde{N} \rightarrow l_{\gamma}^{+} W^{-}, W^{-} \rightarrow j j\right)$ :

Using interactions in (2.21)-(2.22) it is easy to show that the production cross section for this process (after summing over neutrino states $i$ ) is proportional to $\left|K_{\beta}\right|^{2}\left|K_{\gamma}\right|^{2}$. Therefore, as discussed in the previous section, in the narrow width approximation the production cross section for this process and its charge conjugated one can be factorized as

$$
\sigma_{\beta \gamma}^{(1)}\left|\tilde{Y}_{\beta}\right|^{2}\left|\tilde{Y}_{\gamma}\right|^{2}
$$

- $p p \rightarrow E_{2}^{+} \tilde{N}, \quad\left(E_{2}^{+} \rightarrow \nu_{i} W^{+}, W^{+} \rightarrow j j\right) \quad\left(\tilde{N} \rightarrow l_{\beta}^{+} W^{-}, W^{-} \rightarrow l_{\gamma}^{-} \nu_{\gamma}\right)$ : whose production cross section is proportional to $\left|K_{\beta}\right|^{2}\left|\bar{K}_{i}\right|^{2}$. So in the narrow width approximation and after summing over the $\nu_{i}$ states using eq. (2.26) the cross section for this process and its charge conjugated one can be written as

$$
\sigma_{\beta \gamma}^{(2 a)}\left|\tilde{Y}_{\beta}\right|^{2}
$$

- $p p \rightarrow E_{2}^{+} \tilde{N}, \quad\left(E_{2}^{+} \rightarrow \nu_{i} W^{+}, W^{+} \rightarrow l_{\gamma}^{+} \nu_{\gamma}\right) \quad\left(\tilde{N} \rightarrow l_{\beta}^{+} W^{-}, W^{-} \rightarrow j j\right)$ : and the respective charge conjugate process possess a cross section proportional to 
$\left|K_{\beta}\right|^{2}\left|\bar{K}_{i}\right|^{2}$. As before, in the narrow width approximation and after summing over the $\nu_{i}$ states we parametrize the production cross section as

$$
\sigma_{\beta \gamma}^{(2 b)}\left|\tilde{Y}_{\beta}\right|^{2} .
$$

- $p p \rightarrow E_{2}^{+} \tilde{N}, \quad\left(E_{2}^{+} \rightarrow \nu_{i} W^{+}, W^{+} \rightarrow j j\right) \quad\left(\tilde{N} \rightarrow \nu_{j} Z, Z \rightarrow l_{\beta}^{-} l_{\beta}^{+}\right)$:

and its charge conjugate process with cross section proportional to $\left|\bar{K}_{i}\right|^{2}\left|\bar{K}_{j}\right|^{2}$ that after summing over the neutrino states $i$ and $j$ reads

$$
\sigma_{\beta \beta}^{(2 c)} .
$$

- $p p \rightarrow E_{1}^{+} E_{1}^{-}, \quad\left(E_{1}^{+} \rightarrow l_{\beta}^{+} Z, Z \rightarrow j j\right)\left(E_{1}^{-} \rightarrow l_{\gamma}^{-} Z, Z \rightarrow \nu_{i} \nu_{i}\right)$, $p p \rightarrow E_{1}^{+} E_{1}^{-}, \quad\left(E_{1}^{+} \rightarrow l_{\beta}^{+} Z, Z \rightarrow \nu_{i} \nu_{i}\right) \quad\left(E_{1}^{-} \rightarrow l_{\gamma}^{-} Z, Z \rightarrow j j\right)$ : with cross section

$$
\sigma_{\beta \gamma}^{(3)}\left|\tilde{Y}_{\beta}\right|^{2}\left|\tilde{Y}_{\gamma}\right|^{2}
$$

Altogether the cross section of each OS flavour channel is given by:

$$
\begin{aligned}
\sigma_{e e}^{O S} & \equiv\left[\sigma_{e e}^{(1)}+\sigma_{e e}^{(3)}\right]\left|\tilde{Y}_{e}\right|^{4}+\sigma_{e e}^{(2 a)}\left|\tilde{Y}_{e}\right|^{2}+\sigma_{e e}^{(2 c)}, \\
\sigma_{e \mu}^{O S} & \equiv\left[\sigma_{e \mu}^{(1)}+\sigma_{\mu e}^{(1)}+\sigma_{e \mu}^{(3)}+\sigma_{\mu e}^{(3)}\right]\left|\tilde{Y}_{e}\right|^{2}\left|\tilde{Y}_{\mu}\right|^{2}+\sigma_{e \mu}^{(2 a)}\left|\tilde{Y}_{e}\right|^{2}+\sigma_{\mu e}^{(2 a)}\left|\tilde{Y}_{\mu}\right|^{2}, \\
\sigma_{\mu \mu}^{O S} & \equiv\left[\sigma_{\mu \mu}^{(1)}+\sigma_{\mu \mu}^{(3)}\right]\left|\tilde{Y}_{\mu}\right|^{4}+\sigma_{\mu \mu}^{(2 a)}\left|\tilde{Y}_{\mu}\right|^{2}+\sigma_{\mu \mu}^{(2 c)},
\end{aligned}
$$

while for SS lepton final state

$$
\begin{aligned}
\sigma_{e e}^{S S} & \equiv \sigma_{e e}^{(2 b)}\left|\tilde{Y}_{e}\right|^{2}, \\
\sigma_{e \mu}^{S S} & \equiv \sigma_{e \mu}^{(2 b)}\left|\tilde{Y}_{e}\right|^{2}+\sigma_{\mu e}^{(2 b)}\left|\tilde{Y}_{\mu}\right|^{2}, \\
\sigma_{\mu \mu}^{S S} & \equiv \sigma_{\mu \mu}^{(2 b)}\left|\tilde{Y}_{\mu}\right|^{2} .
\end{aligned}
$$

We notice that in eq. (3.1)-(3.7) the $\sigma_{\beta \gamma}^{(1)}, \sigma_{\beta \gamma}^{(2 a)}, \sigma_{\beta \gamma}^{(2 b)}, \sigma_{\beta \gamma}^{(2 c)}$, and $\sigma_{\beta \gamma}^{(3)}$ factors are primarely functions of the heavy mass $M$ and their flavour dependence enters only via the difference response of the $e$ and $\mu$ to the selection cuts. Conversely the normalized yukawas $\tilde{Y}_{\beta}$ contain the dominant dependence on the lepton flavour and are functions of the parameters determined by the neutrino oscillation data and the unknown Majorana phase $\alpha$ give in eq. (2.20).

\subsection{Simulation of the expected event rates}

In our analysis, we first simulated the above parton level signal processes with MadGraph 5 [48]. We then used PYTHIA 6.4 [49] to generate the parton shower and hadronization. Finally we performed a fast detector simulation with DELPHES 3 [50] with jets being reconstructed using the anti- $k_{T}$ algorithm with a radius $R=0.4$ with the package FASTJET [51].

In order to reproduce the ATLAS event selection corresponding to the searches in ref. [22] we required that the events contain exactly two leptons (muons and/or electrons), 
a minimum of two jets and no b-tagged jet. In the case of OS (SS) leptons the leading lepton must have transverse momentum $\left(p_{T}\right)$ in excess of $100(70) \mathrm{GeV}$ with the next-toleading lepton $p_{T}$ larger than $25(40) \mathrm{GeV}$. In addition we imposed the invariant mass of the two leptons to be larger than 130 and $90 \mathrm{GeV}$ for OS and SS events respectively. We also demanded the $p_{T}$ of two leading jets to be larger than 60 (40) and 30 (25) GeV for the OS (SS) final state. Moreover, to characterize the presence of a hadronically decaying $W$ in the event the invariant mass of the leading jet pair was required to be between 60 and $100 \mathrm{GeV}$, and for OS events we require the two leading jets to satisfy $\Delta R_{j j}<2$. Finally, we only selected events presenting missing transverse energy in excess of 110 (100) GeV for OS (SS) events. We implement the above selection in MadAnalysis5 [52].

In order to tune our calculations we first simulated the signal for the Type-III see-saw model of ref. [24] which is the one used in the ATLAS analysis. By comparing our number of expected events with the ones obtained by ATLAS in figure 2 of [22] for the OS and SS final states presenting $e e, e \mu$ and $\mu \mu$ pairs, we extract overall multiplicative correction factors for each of these final states such that our fast simulation agrees with the one of ATLAS. To validate our procedure we verified that our tuned Monte Carlo reproduces the missing transverse momentum distribution presented in figure 1 of [22]. We then apply these correction factors in the evaluation of the expected number of events of the MLFV type-III see-saw model.

Concerning backgrounds, the dominant contribution comes from the production of dibosons ( $W W, W Z, Z Z), Z$ plus jets, top pairs and single top in association with a $W$. In addition there are also background events that stems from misidentification of leptons. In our analysis we directly use the background rates estimated by ATLAS [22].

Figure 2 depicts the resulting cross section factors $\sigma_{1}, \sigma_{2 a}, \sigma_{2 b}$, and $\sigma_{3}$ introduced in eqs. (3.1)-(3.5), for the events passing the selection cuts and after applying the tuning correction factors. The results are shown as a function of the new fermion mass and for the different lepton flavor combinations and a center-of-mass energies of $8 \mathrm{TeV}{ }^{1}$ From this figure we can see that the four possible leptonic final states $(e e, \mu \mu, e \mu$, and $\mu e$ ) have similar cross section factors for masses larger than $300 \mathrm{GeV}$, however, closer to the threshold the detection and acceptance efficiencies induce differences among the leptonic final states. In the case of $\sigma_{2 a}$ both leptons originate from the decay of a single triplet fermion, therefore reducing the acceptance at small masses due to the OS lepton pair invariant mass cut.

Weighting these cross section factors with the different combination of Yukawa couplings as in eqs. (3.6) and (3.7) and times the luminosity we predict the signal event rates in the different flavour and charge combinations of the final lepton pair. For example in figures 3 and 4 we present the event rate prediction as a function of the unknown Majorana phase $\alpha$ for a triplet mass of $300 \mathrm{GeV}$, an integrated luminosity of $20.3 \mathrm{fb}^{-1}$ and normal ordering and inverted ordering respectively. As seen in figure 3 for NO, the expected number of ee events is very small for both OS and SS channels. Nevertheless, a considerable number of events containing muons is expected except for $\alpha / \pi$ around 1 . This is so because for NO $\left|\tilde{Y}_{\mu}\right|$ is much larger than $\left|\tilde{Y}_{e}\right|$ as can be seen from figure 1. For IO we see in figure 4, as could be anticipated from figure 1 , that the expectations in all flavor channels vary

\footnotetext{
${ }^{1} \sigma_{2 c}$ is vanishing small since the event selection vetoes the presence of on-shell $Z$ 's.
} 

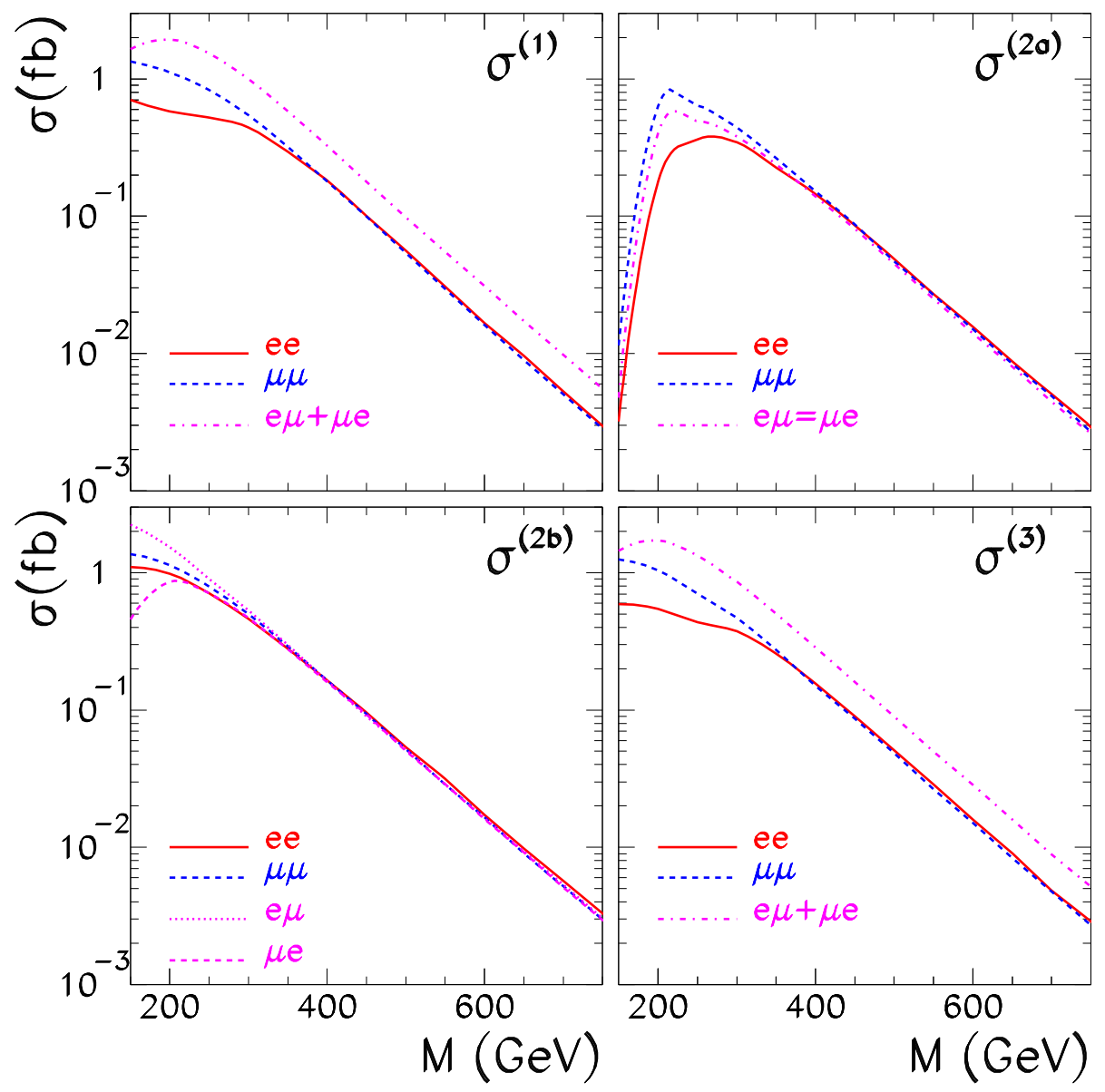

Figure 2. Cross section factors for the different contributions to the event topologies as a function of the triplet mass $M$ as defined in eqs. (3.1)-(3.5) after inclusion of our simulation of the selections of ref. [22] (see text for details).

appreciably with the Majorana phase $\alpha$. However, the strong correlation between $\left|\tilde{Y}_{e}\right|$ and $\left|\tilde{Y}_{\mu}\right|$ in IO - depicted in the right bottom panel of figure 1 - guarantees a sizable number of events for almost the entire $\alpha$ range with the signal being dominated by the channels $e \mu$ and $\mu \mu$ for OS leptons and the channel $e \mu$ for SS leptons.

\section{Analysis: results and discussion}

In order to quantify the bounds on the MLFV Type-III see-saw scenario we build the likelihood function using the six data points associated to the events with $e e, e \mu$ and $\mu \mu$ leptons of either SS or OS. As discussed in the previous section, we make direct use of the corresponding background estimate by ATLAS which we read from figure 2 and table I of ref. [22] and that summarize here for completeness:

\begin{tabular}{|l|cccccc|cc|}
\hline & OS $e e$ & SS $e e$ & OS $\mu \mu$ & SS $\mu \mu$ & OS $e \mu$ & SS $e \mu$ & total OS & total SS \\
\hline$N^{\text {dat }}$ & 9 & 3 & 3 & 1 & 13 & 0 & 25 & 4 \\
$N^{\text {bck }}$ & 8.5 & 1 & 9.5 & 0.5 & 13 & 1.65 & $31.0 \pm 7.7$ & $3.15 \pm 0.8$ \\
\hline
\end{tabular}




\section{NO $M=300 \mathrm{GeV}$}
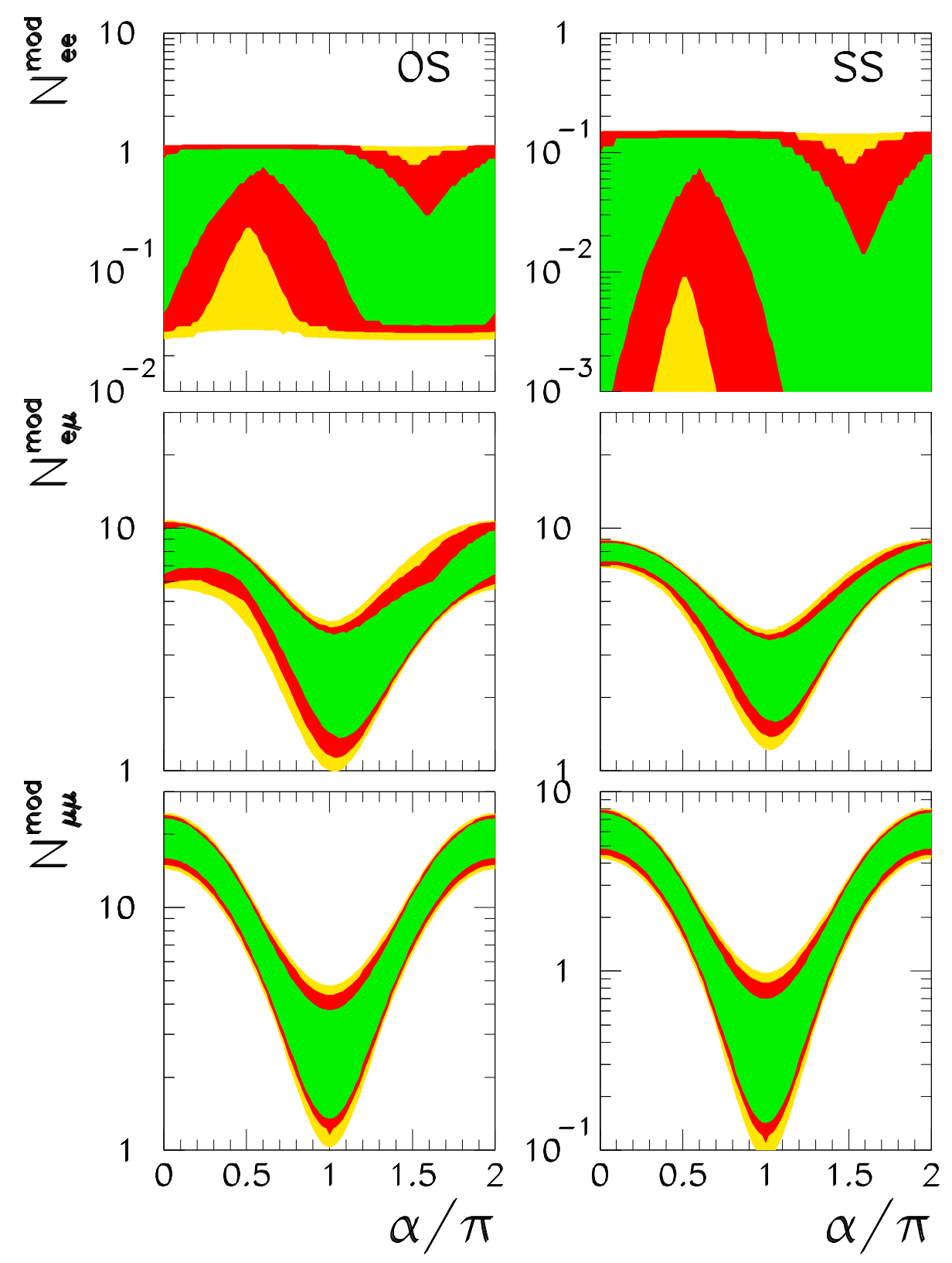

Figure 3. Expected number of signal events at $\sqrt{s}=8 \mathrm{TeV}$ and an integrated luminosity of $20.3 \mathrm{fb}^{-1}$, in each of the six flavour-charge combinations for a triplet mass of $M=300 \mathrm{GeV}$ and for neutrino parameters corresponding to the NO. The conventions are the same as in figure 1.

According to ref. [22] the reported background errors in the table and figure include both the statistical and systematic uncertainties. Comparing the read out of these uncertainties for each of the six individual channels with the total reported in the table we conclude that the $\sim 25 \%$ background uncertainty is strongly correlated among the different channels as the total background uncertainty comes to be very close to the arithmetic sum of the 


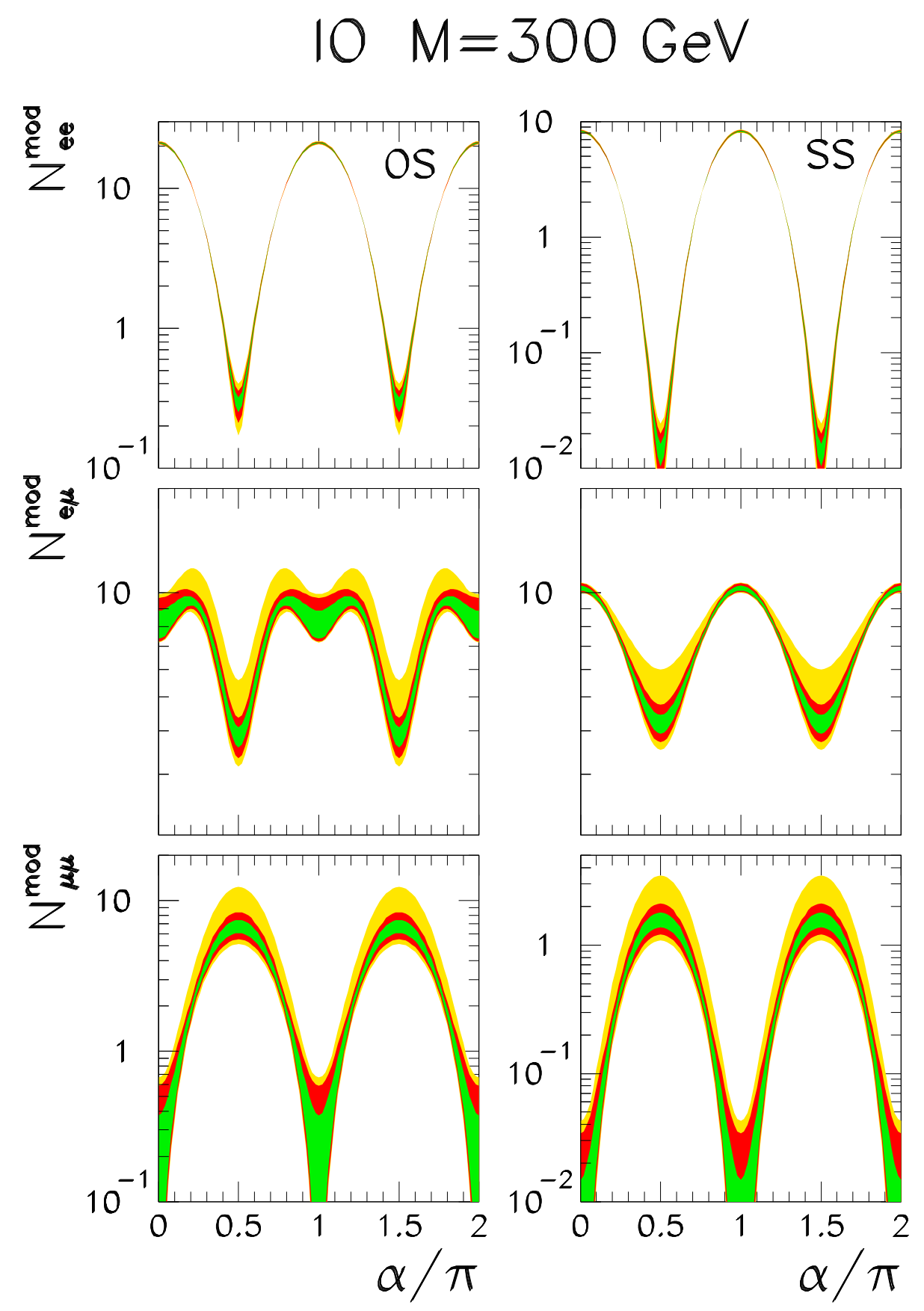

Figure 4. Same as figure 4 for IO.

individual ones, while if they were totally uncorrelated one would expect it to be the quadratic sum.

So we build the likelihood function as

$$
-2 \mathcal{L}_{6 d}=\chi_{6 d}^{2}=\min _{\xi}\left\{2 \sum_{i=1,6}\left[(1+\xi) N_{i}^{\mathrm{bck}}+N_{i}^{\mathrm{mod}}-N_{i}^{\mathrm{dat}} \log \frac{N_{i}^{\mathrm{dat}}}{\left(N_{i}^{\mathrm{bck}}+N_{i}^{\mathrm{mod}}\right)}\right]+\frac{\xi^{2}}{0.25^{2}}\right\}
$$




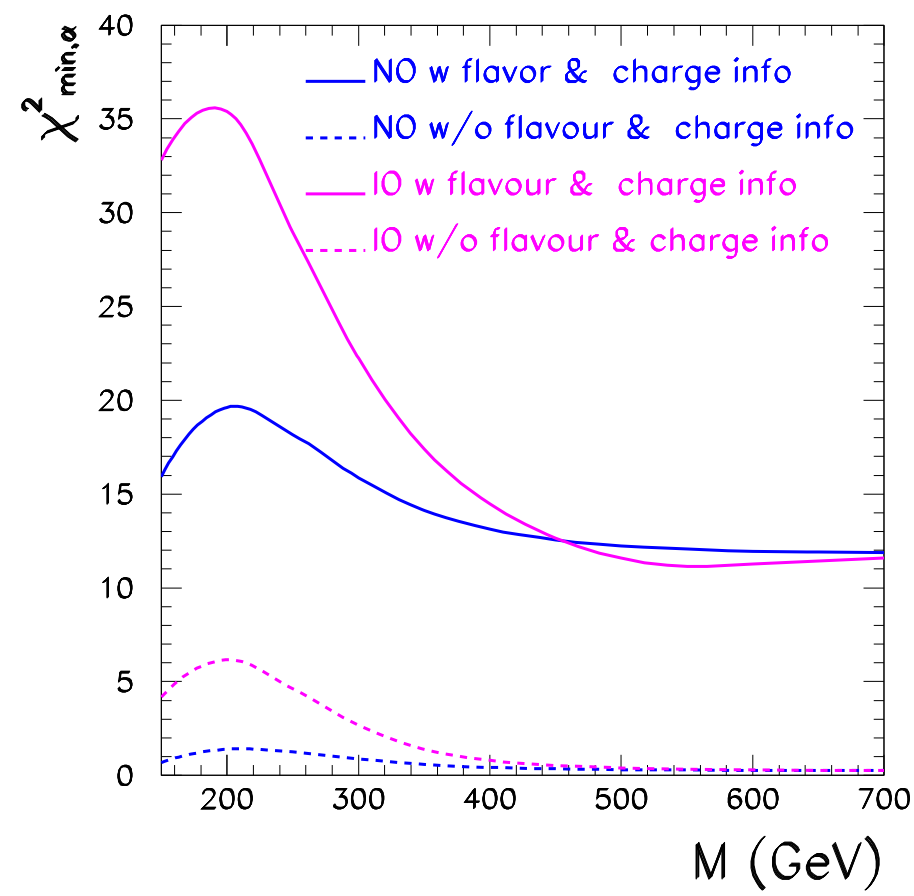

Figure 5. Triplet mass dependence of the $\chi^{2}$ functions of the analysis of $p p \rightarrow l l^{\prime} j j \nu \nu$ events with $l, l^{\prime}$ being either $e$ or $\mu$ of either charge observed in the LHC Run-I in ATLAS [22] when analyzed in the context of MLFV Type-III see-saw model. The full lines correspond to the likelihood function constructed including the full information given on flavour and charge of the final states (see eq. (4.1)) while the dashed lines are obtained from the analysis of the total data summing over charge and flavour. The triplet couplings have been marginalized within the ranges allowed at $95 \%$ CL by the neutrino oscillation data analysis in ref. [35] for NO (blue lines) and IO (purple lines) and for any value of the Majorana phase $\alpha$.

where we account for the background uncertainty by introducing a unique pull $\xi$ with an uncertainty of $25 \%{ }^{2} N_{i}^{\text {mod }}$ is the predicted contribution to the number of events in channel $i$ from the triplet production and decays which depends on the triplet mass and the neutrino parameters as discussed in the previous section. In building the likelihood (4.1) we have used Poisson statistics to account for the small number of events in each channel.

We plot as full lines in figure 5 the dependence of $\chi_{6 d}^{2}$ on the triplet mass after marginalization over the neutrino parameters (including the unknown Majorana phase $\alpha$ ) over the 95\% CL allowed values from the neutrino oscillation analysis for either NO or IO. First thing to notice is that in the $\mathrm{SM}\left(N_{i}^{\mathrm{mod}}=0\right.$, which can be inferred from the large M limit in the figure) we find $\chi_{6 d, \mathrm{SM}}^{2}=11.8$ which is a bit high for 6 data points. This is mostly driven by the OS $\mu \mu$ channel for which 3 events are observed when about 10 are expected in the SM. From this figure we also read that requiring $\chi_{6 d}^{2}-\chi_{6 d, \mathrm{SM}}^{2}<4$ we can infer an absolute bound on the triplet mass of $300 \mathrm{GeV}(375 \mathrm{GeV})$ for NO (IO) light neutrino masses.

\footnotetext{
${ }^{2}$ We have verified that including several pulls for the different source of background and smaller uncertainties each does not have any significant impact in the results.
} 
In order to stress the importance of the flavour and charge information on the possibility of imposing this bound, we have constructed the corresponding likelihood function summing the information from all the channels. As in this case the total number of observed events is large enough, we assume gaussianity. So we define:

$$
\chi_{\text {tot }}^{2}=\frac{\left(N^{\text {tot,bck }}+N^{\text {tot,mod }}-N^{\text {tot,dat }}\right)^{2}}{N^{\text {tot,dat }}+\left(0.25 N^{\text {tot,bck }}\right)^{2}} .
$$

The dependence of $\chi_{\text {tot }}^{2}$ on the triplet mass after marginalization over the neutrino parameters (including the unknown Majorana phase $\alpha$ ) over the 95\% CL allowed values from the neutrino oscillation analysis $\left(\Delta \chi_{\text {osc }}^{2} \leq 4\right)$ for either NO or IO is shown as dashed lines in figure 5. As the deficit in OS $\mu \mu$ is compensated by the slight excesses in other channels, we find that in this case the SM gives a perfect description of the total observed event rates $\left(\chi_{\mathrm{tot}, \mathrm{SM}}^{2}=0.25\right)$. The figure clearly illustrates the relevance of flavour and charge information, as in this case the condition $\chi_{\text {tot }}^{2}-\chi_{\text {tot,SM }}^{2}<4$ does result into no bound on the triplet mass for NO neutrino masses while it rules out only $M<260$ for IO.

The dependence of the bounds on the unknown phase $\alpha$ is displayed in figure 6 . The full red regions are excluded values of triplet masses in the MLFV scenario at 95\% CL $\left(\chi_{6 d}^{2}-\chi_{6 d, \mathrm{SM}}^{2}>4\right)$ when marginalizing over the oscillation parameters within the $95 \% \mathrm{CL}$ allowed values by the oscillation analysis in NO (left) and IO (right) for each value of $\alpha$. The $\alpha$ marginalized bound discussed above correspond to the lightest allowed masses in these panels which for $\mathrm{NO}(M>300 \mathrm{GeV})$ correspond to $\alpha=\pi$ while for $\alpha=0,2 \pi$ the bound strengthens to $M>480 \mathrm{GeV}$. For IO the dependence of the bound on $\alpha$ is weaker. The marginalized bound $M>380 \mathrm{GeV}$ corresponds to $\alpha \sim 3 \pi / 4,3 \pi / 2$ but it is close to that value for almost all values of alpha. The strongest bound of $M>430 \mathrm{GeV}$ corresponds also to $\alpha=0,2 \pi$.

The hatched regions are the corresponding constraints obtained using only the information on the total number of events $\left(\chi_{\text {tot }}^{2}-\chi_{\text {tot,SM }}^{2}>4\right)$ summed over flavour and charge of the final leptons. This figure illustrates again how using the flavour and charge information allows to impose stronger bounds on this scenario, in particular allowing to rule out triplet masses irrespective of $\alpha$ for both orderings while for NO no bound can be imposed for $70^{\circ} \lesssim \alpha \lesssim 250^{\circ}$ if only the total number of events is considered.

In summary, we have shown how the analysis of the events containing two charged leptons (either electron or muons), two jets from a hadronically decaying $W$ boson in the Run I with the ATLAS detector [22] can be used to impose constraints on the MLFV Type III see-saw scenario. Because of MLFV, the expected event rates in the different flavour and charge combinations of the two leptons are constrained by the existing neutrino data so the bounds cannot be evaded. For this reason it is possible to use this data to rule out these scenario with triplet masses lighter than $300 \mathrm{GeV}$ at $95 \%$ CL irrespective of the neutrino mass ordering and of the value of the unknown Majorana phase parameter. The same analysis allows to rule out triplet masses up to $480 \mathrm{GeV}$ at $95 \%$ CL for $\mathrm{NO}$ and $\alpha=0, \pi$. We have stressed and quantified how the information on flavour and charge information of the produced leptons is important for maximal sensitivity to MLFV. In particular we 


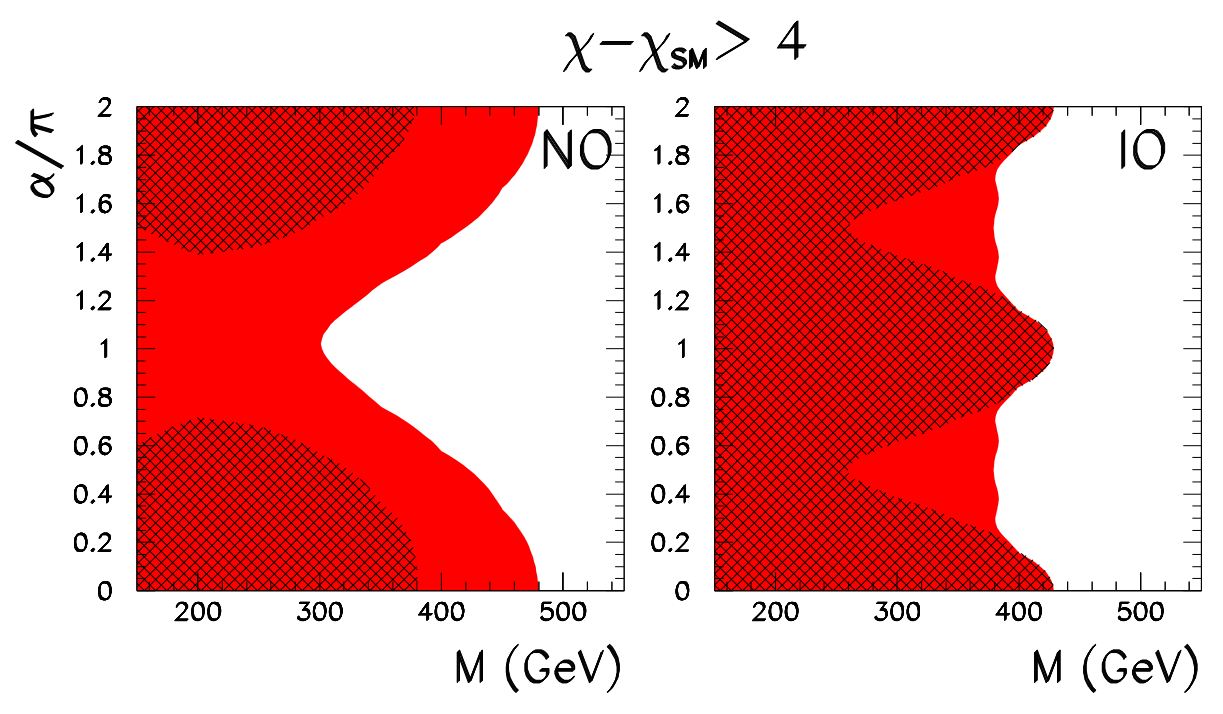

Figure 6. $95 \%$ excluded triplet mass in the MLFV Type-III see-saw scenario as a function of the unknown phase $\alpha$ from the analysis of $p p \rightarrow l l^{\prime} j j \nu \nu$ events with $l, l^{\prime}$ being either $e$ or $\mu$ of either charge observed in the LHC Run-I in ATLAS [22]. The full regions correspond to the likelihood function constructed including the full information given on flavour and charge of the final states (see eq. (4.1)) while the hatched ones are obtained from the analysis of the total data summing over charge and flavour (see eq. (4.2)). The triplet couplings have been marginalized within the ranges allowed at 95\% CL by the neutrino oscillation data analysis in ref. [35] for NO (left) and IO (right).

showed that in the absence of information on charge and flavour of the final leptons it is not possible to obtain unambiguous limits on the mass of the new triplet fermions.

We finish by commenting that extended sensitivity to MLFV with heavier triplets should be attainable with the data already accumulated from Run II in the same or other event topologies. For example by the analysis of the multilepton final states in CMS in ref. [19] which, so far, has been performed only in the context of the simplified Type-III see-saw model. Nevertheless, as previously stressed to do so it is important to make use of the flavour and charge of final state leptons which has not been made public yet. To this aim, we have made available the model files for the MLFV Type-III see-saw [47].

\section{Acknowledgments}

M.C.G-G wants to thank her NUFIT collaborators, I. Esteban, M. Maltoni, I. Martinez and T. Schwetz for their generous contribution of the results from the data oscillation analysis used in this article. She also wants to thank the USP group for their hospitality during the final stages of this work. This work is supported in part by Conselho Nacional de Desenvolvimento Científico e Tecnológico (CNPq) and by Fundação de Amparo à Pesquisa do Estado de São Paulo (FAPESP) grants 2012/10095-7 and 2017/06109-5, by U.S.A.-NSF grant PHY-1620628, by EU Networks FP10 ITN ELUSIVES (H2020-MSCA-ITN-2015-674896) and INVISIBLES-PLUS (H2020-MSCA-RISE-2015-690575), by MINECO grant FPA201676005-C2-1-P and by Maria de Maetzu program grant MDM-2014-0367 of ICCUB. 
Open Access. This article is distributed under the terms of the Creative Commons Attribution License (CC-BY 4.0), which permits any use, distribution and reproduction in any medium, provided the original author(s) and source are credited.

\section{References}

[1] M.C. Gonzalez-Garcia and M. Maltoni, Phenomenology with Massive Neutrinos, Phys. Rept. 460 (2008) 1 [arXiv:0704.1800] [INSPIRE].

[2] S. Weinberg, Baryon and Lepton Nonconserving Processes, Phys. Rev. Lett. 43 (1979) 1566 [INSPIRE].

[3] P. Minkowski, $\mu \rightarrow$ er at a Rate of One Out of $10^{9}$ Muon Decays?, Phys. Lett. B 67 (1977) 421 [INSPIRE].

[4] T. Yanagida, Horizontal Symmetry And Masses Of Neutrinos, Conf. Proc. C 7902131 (1979) 95 [INSPIRE].

[5] M. Gell-Mann, P. Ramond and R. Slansky, Complex Spinors and Unified Theories, Conf. Proc. C 790927 (1979) 315 [arXiv: 1306.4669] [InSPIRE].

[6] R.N. Mohapatra and G. Senjanović, Neutrino Mass and Spontaneous Parity Violation, Phys. Rev. Lett. 44 (1980) 912 [INSPIRE].

[7] W. Konetschny and W. Kummer, Nonconservation of Total Lepton Number with Scalar Bosons, Phys. Lett. B 70 (1977) 433 [INSPIRE].

[8] T.P. Cheng and L.-F. Li, Neutrino Masses, Mixings and Oscillations in $\mathrm{SU}(2) \times \mathrm{U}(1)$ Models of Electroweak Interactions, Phys. Rev. D 22 (1980) 2860 [inSPIRE].

[9] G. Lazarides, Q. Shafi and C. Wetterich, Proton Lifetime and Fermion Masses in an $\mathrm{SO}(10)$ Model, Nucl. Phys. B 181 (1981) 287 [InSPIRE].

[10] J. Schechter and J.W.F. Valle, Neutrino Masses in $\mathrm{SU}(2) \times \mathrm{U}(1)$ Theories, Phys. Rev. D 22 (1980) 2227 [INSPIRE].

[11] R.N. Mohapatra and G. Senjanović, Neutrino Masses and Mixings in Gauge Models with Spontaneous Parity Violation, Phys. Rev. D 23 (1981) 165 [inSPIRE].

[12] R. Foot, H. Lew, X.G. He and G.C. Joshi, Seesaw Neutrino Masses Induced by a Triplet of Leptons, Z. Phys. C 44 (1989) 441 [inSPIRE].

[13] R.N. Mohapatra and J.W.F. Valle, Neutrino Mass and Baryon Number Nonconservation in Superstring Models, Phys. Rev. D 34 (1986) 1642 [INSPIRE].

[14] J. Kersten and A.Y. Smirnov, Right-Handed Neutrinos at CERN LHC and the Mechanism of Neutrino Mass Generation, Phys. Rev. D 76 (2007) 073005 [arXiv:0705.3221] [InSPIRE].

[15] M.-C. Chen and J. Huang, TeV Scale Models of Neutrino Masses and Their Phenomenology, Mod. Phys. Lett. A 26 (2011) 1147 [arXiv:1105.3188] [INSPIRE].

[16] F. del Aguila and J.A. Aguilar-Saavedra, Distinguishing seesaw models at LHC with multi-lepton signals, Nucl. Phys. B 813 (2009) 22 [arXiv:0808.2468] [INSPIRE].

[17] J.A. Aguilar-Saavedra, P.M. Boavida and F.R. Joaquim, Flavored searches for type-III seesaw mechanism at the LHC, Phys. Rev. D 88 (2013) 113008 [arXiv:1308.3226] [INSPIRE]. 
[18] CMS collaboration, Search for heavy lepton partners of neutrinos in proton-proton collisions in the context of the type-III seesaw mechanism, Phys. Lett. B 718 (2012) 348 [arXiv: 1210.1797] [INSPIRE].

[19] CMS collaboration, Search for evidence of Type-III seesaw mechanism in multilepton final states in pp collisions at $\sqrt{s}=13 \mathrm{TeV}$, CMS-PAS-EXO-17-006 (2017) [INSPIRE].

[20] CMS collaboration, Search for Type-III Seesaw Heavy Fermions with Multilepton Final States using $2.3 \mathrm{fb}^{-1}$ of $13 \mathrm{TeV}$ proton-proton Collision Data, CMS-PAS-EXO-16-002 (2016) [INSPIRE].

[21] CMS collaboration, Search for heavy lepton partners of neutrinos in pp collisions at $8 \mathrm{TeV}$, in the context of type-III seesaw mechanism, CMS-PAS-EXO-14-001 (2015) [INSPIRE].

[22] ATLAS collaboration, Search for type-III Seesaw heavy leptons in pp collisions at $\sqrt{s}=8$ TeV with the ATLAS Detector, Phys. Rev. D 92 (2015) 032001 [arXiv:1506.01839] [INSPIRE].

[23] ATLAS collaboration, Search for Type III Seesaw Model Heavy Fermions in Events with Four Charged Leptons using $5.8 \mathrm{fb}^{-1}$ of $\sqrt{\mathrm{s}}=8 \mathrm{TeV}$ data with the ATLAS Detector, ATLAS-CONF-2013-019 (2013) [INSPIRE].

[24] C. Biggio and F. Bonnet, Implementation of the Type III Seesaw Model in FeynRules/MadGraph and Prospects for Discovery with Early LHC Data, Eur. Phys. J. C 72 (2012) 1899 [arXiv:1107.3463] [INSPIRE].

[25] R.S. Chivukula and H. Georgi, Composite Technicolor Standard Model, Phys. Lett. B 188 (1987) 99 [INSPIRE].

[26] A.J. Buras, P. Gambino, M. Gorbahn, S. Jager and L. Silvestrini, Universal unitarity triangle and physics beyond the standard model, Phys. Lett. B 500 (2001) 161 [hep-ph/0007085] [INSPIRE].

[27] G. D’Ambrosio, G.F. Giudice, G. Isidori and A. Strumia, Minimal flavor violation: An Effective field theory approach, Nucl. Phys. B 645 (2002) 155 [hep-ph/0207036] [INSPIRE].

[28] V. Cirigliano, B. Grinstein, G. Isidori and M.B. Wise, Minimal flavor violation in the lepton sector, Nucl. Phys. B 728 (2005) 121 [hep-ph/0507001] [INSPIRE].

[29] S. Davidson and F. Palorini, Various definitions of Minimal Flavour Violation for Leptons, Phys. Lett. B 642 (2006) 72 [hep-ph/0607329] [INSPIRE].

[30] M.B. Gavela, T. Hambye, D. Hernández and P. Hernández, Minimal Flavour Seesaw Models, JHEP 09 (2009) 038 [arXiv:0906.1461] [INSPIRE].

[31] R. Alonso, G. Isidori, L. Merlo, L.A. Muñoz and E. Nardi, Minimal flavour violation extensions of the seesaw, JHEP 06 (2011) 037 [arXiv:1103.5461] [INSPIRE].

[32] P. Fileviez Perez, T. Han, G.-y. Huang, T. Li and K. Wang, Neutrino Masses and the CERN LHC: Testing Type II Seesaw, Phys. Rev. D 78 (2008) 015018 [arXiv:0805.3536] [InSPIRE].

[33] J. Garayoa and T. Schwetz, Neutrino mass hierarchy and Majorana CP phases within the Higgs triplet model at the LHC, JHEP 03 (2008) 009 [arXiv:0712.1453] [INSPIRE].

[34] O.J.P. Éboli, J. Gonzalez-Fraile and M.C. Gonzalez-Garcia, Neutrino Masses at LHC: Minimal Lepton Flavour Violation in Type-III See-saw, JHEP 12 (2011) 009 [arXiv:1108.0661] [INSPIRE]. 
[35] I. Esteban, M.C. Gonzalez-Garcia, M. Maltoni, I. Martinez-Soler and T. Schwetz, Updated fit to three neutrino mixing: exploring the accelerator-reactor complementarity, JHEP 01 (2017) 087 [arXiv: 1611.01514] [INSPIRE].

[36] A. Abada, C. Biggio, F. Bonnet, M.B. Gavela and T. Hambye, Low energy effects of neutrino masses, JHEP 12 (2007) 061 [arXiv:0707.4058] [INSPIRE].

[37] J. Schechter and J.W.F. Valle, Comment on the Lepton Mixing Matrix, Phys. Rev. D 21 (1980) 309 [INSPIRE].

[38] S. Antusch, C. Biggio, E. Fernandez-Martinez, M.B. Gavela and J. Lopez-Pavon, Unitarity of the Leptonic Mixing Matrix, JHEP 10 (2006) 084 [hep-ph/0607020] [INSPIRE].

[39] R. Franceschini, T. Hambye and A. Strumia, Type-III see-saw at LHC, Phys. Rev. D 78 (2008) 033002 [arXiv:0805.1613] [INSPIRE].

[40] B. Bajc, M. Nemevšek and G. Senjanović, Probing seesaw at LHC, Phys. Rev. D 76 (2007) 055011 [hep-ph/0703080] [INSPIRE].

[41] F. del Aguila and J.A. Aguilar-Saavedra, Electroweak scale seesaw and heavy Dirac neutrino signals at LHC, Phys. Lett. B 672 (2009) 158 [arXiv:0809.2096] [InSPIRE].

[42] ATLAS collaboration, The ATLAS Experiment at the CERN Large Hadron Collider, 2008 JINST 3 S08003 [INSPIRE].

[43] CMS collaboration, The CMS Experiment at the CERN LHC, 2008 JINST 3 S08004 [INSPIRE].

[44] T. Li and X.-G. He, Neutrino Masses and Heavy Triplet Leptons at the LHC: Testability of Type III Seesaw, Phys. Rev. D 80 (2009) 093003 [arXiv:0907.4193] [InSPIRE].

[45] N.D. Christensen and C. Duhr, FeynRules - Feynman rules made easy, Comput. Phys. Commun. 180 (2009) 1614 [arXiv:0806.4194] [INSPIRE].

[46] A. Alloul, N.D. Christensen, C. Degrande, C. Duhr and B. Fuks, FeynRules 2.0 - A complete toolbox for tree-level phenomenology, Comput. Phys. Commun. 185 (2014) 2250 [arXiv: 1310.1921] [INSPIRE].

[47] http://feynrules.irmp.ucl.ac.be/wiki.

[48] J. Alwall et al., The automated computation of tree-level and next-to-leading order differential cross sections and their matching to parton shower simulations, JHEP 07 (2014) 079 [arXiv: 1405.0301] [INSPIRE].

[49] T. Sjöstrand, S. Mrenna and P.Z. Skands, PYTHIA 6.4 Physics and Manual, JHEP 05 (2006) 026 [hep-ph/0603175] [INSPIRE].

[50] DELPHES 3 collaboration, J. de Favereau et al., DELPHES 3: a modular framework for fast simulation of a generic collider experiment, JHEP 02 (2014) 057 [arXiv:1307.6346] [INSPIRE].

[51] M. Cacciari, G.P. Salam and G. Soyez, FastJet User Manual, Eur. Phys. J. C 72 (2012) 1896 [arXiv: 1111.6097] [INSPIRE].

[52] E. Conte, B. Fuks and G. Serret, MadAnalysis 5, A User-Friendly Framework for Collider Phenomenology, Comput. Phys. Commun. 184 (2013) 222 [arXiv:1206.1599] [INSPIRE]. 